\title{
Subthreshold Laser Therapy for the Treatment of Refractory Diabetic Macular Edema: A Case Report
}

\author{
Alejandro L Perez ${ }^{1,2}$, Antonio Yaghy ${ }^{2}$ and Andres Emanuelli ${ }^{1,3,4 *}$ \\ ${ }^{1}$ University of Puerto Rico School of Medicine \\ ${ }^{2}$ Wills Eye Hospital, Philadelphia, PA, USA \\ ${ }^{3}$ Retina Care, Arecibo, Puerto Rico \\ ${ }^{4}$ Emanuelli Research and Development Center, Arecibo, Puerto Rico \\ *Corresponding Author: Andres Emanuelli, University of Puerto Rico School of Medicine.
}

Received: September 19, 2019; Published: September 24, 2019

DOI: 10.31080/ASMS.2019.03.0416

\begin{abstract}
Diabetic macular edema is a common complication of diabetic retinopathy that causes loss of visual acuity. It becomes more challenging to treat when it involves the center of the macula. Monthly intravitreal injections are a burden for patients and focal photocoagulation laser has limitations that inhibit its use over the macula. Subthreshold laser therapy has been shown effective in treating mild diabetic macular edema. This case reports the successful treatment of moderate center involved diabetic macular edema with subthreshold laser therapy.
\end{abstract}

Keywords: Diabetic Macular Edema; Subthreshold Laser Therapy

\section{Introduction}

Diabetic retinopathy is one of the leading causes of visual acuity loss worldwide [1]. The main cause of visual impairment in these patients is diabetic macular edema (DME), which can occur at any stage of the disease [2]. The role of inflammation has been suggested to be a key factor in the development of DME [3]. For many years, focal laser photocoagulation had been the gold standard for management of DME to reduce the risk of vision loss [4,5]. However, anti-vascular endothelial growth factors (VEGF) agents have been established to result in better visual acuity in patients with DME than those treated exclusively with laser photocoagulation [6-8].

In recent studies, subthreshold laser therapy (SL) has been shown to be effective in the treatment of mild DME [9]. It is a safer alternative to laser photocoagulation since it does not cause structural damage to the underlying photoreceptor layer and prevents retinal scars, allowing both anatomical and functional preservation of the retina $[10,11]$.

Herein, we report a case of DME refractory to intravitreal bevacizumab and focal laser photocoagulation (FOCAL).

\section{Case Report}

A 63-year-old Hispanic female with a history of diabetes mellitus (DM) presented with blurred vision, more significant in the left eye (OS) than the right eye (OD). The vision had been worsening progressively for several months until she decided to seek treatment.

On examination, best-corrected visual acuity (BCVA) was 20/100 in both eyes (OU) and intraocular pressure was $25 \mathrm{~mm}$ Hg OU. Slit lamp examination showed no abnormal findings OU. Dilated fundus examination was remarkable for background diabetic retinopathy and center involved macular edema (CI-DME) OU 
(Figure 1A and 1B). Macular optical coherence tomography (OCT) showed moderate CI-DME with a central macular thickness (CMT) of $598 \mu \mathrm{m}$ at presentation (Figure 2A).

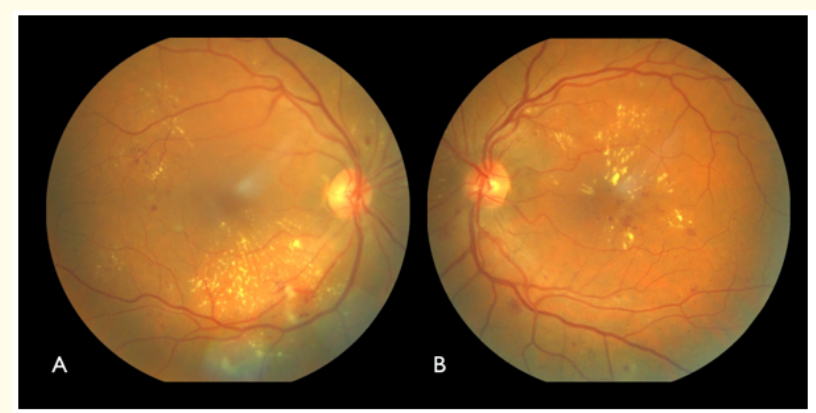

Figure 1: 63-year-old Hispanic female with a history of diabetes mellitus (DM) presented with blurred vision OU. (A) Fundus photo OD and OS (B) showed background diabetic retinopathy and center involved diabetic macular edema (CI-DME).

The patient was treated with an intravitreal bevacizumab injection and a session of FOCAL to the peripheral retina OU at initial presentation, followed by another session of FOCAL 2 months later. This resulted in the improvement of the CI-DME and an increase in the BCVA to 20/50 OD. However, the left eye failed to show a significant response to treatment at 2-month follow-up (Figure 2B). Combinations of intravitreal bevacizumab and FOCAL were used to treat the patient for the following 2 years, but persistent CI-DME was observed with CMT of $406 \mu \mathrm{m}$ (Figure 2C). Subthreshold laser therapy was then added to the treatment regimen OS. This was done with the Easy Ret ${ }^{\circledR} 577 \mathrm{~nm}$ Laser by Quantel Medical in the subliminal laser pattern mode. This treatment resulted in dramatic improvement of the CI-DME with CMT of $237 \mu \mathrm{m}$ on OCT 3 months after SL therapy (Figure 2D). BCVA also improved to 20/30 OS.

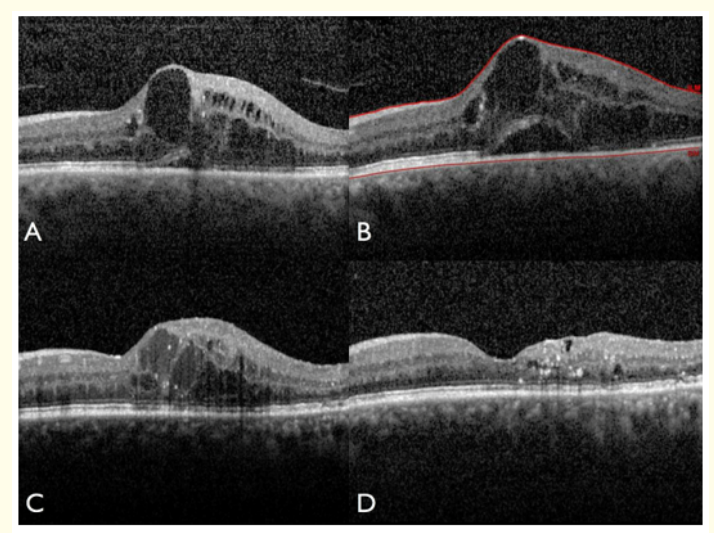

Figure 2: (A) Optical coherence tomography (OCT) of the left eye revealed CI-DME. (B) Treatment with monthly intravitreal injections for 2 months and 2 sessions of FOCAL failed to resolve the CI-DME. (C) Despite continuation of the monthly intravitreal injections and FOCAL sessions for 2 years, the CI-DME did not resolve. (D) Application of subthreshold laser therapy resulted in dramatic resolution of CI-DME.

\section{Discussion}

In recent years, new management options to treat DME emerged. This is due to the fact that, even though intravitreal anti-VEGF is an effective treatment that results in improvement of vision, the possible lifetime duration of the therapy is incompatible with most of the patient's lifestyle as monthly injections can become a heavy burden for patients on the long term [12].

In addition, the use of conventional FOCAL treatment is usually avoided over the macula to prevent visual loss as it can result in damage of the outer retina and subsequent scar formation. Although the mechanism is unknown, it has been proposed that the laser scars result in apoptosis of the photoreceptors, retinal pigment epithelium, and choriocapillaris [13]. However, the inner retina is relieved of hypoxia due to the fact that the scar allows oxygen to diffuse through it [13].

A study by Chhablani., et al. in 2018 showed SL to be safe and effective in achieving better anatomical, visual, and functional outcomes in the treatment of DME [14]. This and other studies have encouraged retina specialists to incorporate SL as a monotherapy or as an adjuvant to anti-VEGF in the treatment of DME.

This patient showed significant improvement in BCVA when SL was incorporated in the management for her moderate CI-DME. SL was applied to the macular region without fear of complications since it does not cause structural damage to the underlying photoreceptor layer and prevents retinal scars, allowing both anatomical and functional preservation of the retina $[10,11]$. SL demonstrated significant improvement of moderate CI-DME and vision in this patient that was maintained 8 months after treatment.

\section{Conclusion}

Frequent anti-VEGF injections for the treatment of CI-DME can be a burden for patients. Newer management options, such as SL, may decrease this burden in refractory cases. This case demonstrated SL therapy to be successful in managing moderate CI-DME, resulting in a significant increase in BCVA as well.

\section{Bibliography}

1. Flaxman SR., et al. "Global causes of blindness and distance vision impairment 1990-2020: a systematic review and metaanalysis". Lancet Glob Heal 5.12 (2017): e1221-e1234.

2. Michaelides M., et al. "A Prospective Randomized Trial of Intravitreal Bevacizumab or Laser Therapy in the Management of Diabetic Macular Edema (BOLT Study). 12-Month Data: Report 2". Ophthalmology 117.6 (2010): 1078-1086.

3. Lee H., et al. "Association between soluble cd14 in the aqueous humor and hyperreflective foci on optical coherence tomography in patients with diabetic macular edema". Investigative ophthalmology and visual science 59.2 (2018): 715-721.

4. Bhagat N., et al. "Diabetic Macular Edema: Pathogenesis and Treatment". Survey of ophthalmology 54.21 (2009): 1-32. 
5. Barkmeier AJ., et al. "Effectiveness of laser photocoagulation in clinically significant macular edema with focal versus diffuse parafoveal thickening on optical coherence tomography". Ophthalmic Surgery, Lasers and Imaging 40.5 (2009): 472479.

6. Nguyen QD., et al. "Two-year outcomes of the ranibizumab for edema of the mAcula in diabetes (READ-2) study". Ophthalmology 117.11 (2010): 2146-2151.

7. Korobelnik JF., et al. "Intravitreal aflibercept for diabetic macular edema”. Ophthalmology 121.11 (2014): 2247-2254.

8. Arevalo JF., et al. "Primary Intravitreal Bevacizumab for Diffuse Diabetic Macular Edema. The Pan-American Collaborative Retina Study Group at 24 Months". Ophthalmology 116.8 (2009): 1488-1497.

9. Citirik M. "The impact of central foveal thickness on the efficacy of subthreshold micropulse yellow laser photocoagulation in diabetic macular edema". Lasers in medical science 34.5 (2019): 907-912.

10. Wells-Gray EM., et al. "Structural integrity of individual cone photoreceptors after short-wavelength subthreshold micropulse laser therapy for diabetic macular edema". Ophthalmic Surg Lasers Imaging Retina 49.12 (2018): 946-954.

11. K Luttrull J and Dorin G. "Subthreshold Diode Micropulse Laser Photocoagulation (SDM) as Invisible Retinal Phototherapy for Diabetic Macular Edema: A Review". Current Diabetes Reviews 8.4 (2012): 274-284.

12. Ford J., et al. "Current treatments in diabetic macular oedema: Systematic review and meta-analysis". BMJ Open (2013).

13. Chhablani J., et al. "Comparison of different settings for yellow subthreshold laser treatment in diabetic macular edema". BMC Ophthalmology 18 (2018): 168.

14. Stefánsson E. "The therapeutic effects of retinal laser treatment and vitrectomy. A theory based on oxygen and vascular physiology". Acta Ophthalmol Scand 79.5 (2001): 435-440.

\section{Volume 3 Issue 10 October 2019}

(C) All rights are reserved by Andres Emanuelli., et al. 